\title{
EFFECT OF A TRAINING MACROCYCLE ON PHYSIOLOGICAL INDICATORS, BODY COMPOSITION, AND EXPLOSIVENESS AMONG RECREATIONAL RUNNERS
}

original paper

( ) University School of Physical Education in Wroclaw

DOI: https://doi.org/10.5114/hm.2020.88153

\author{
KARINA ALVES DA SILVA ${ }^{1,2}$, JULIANA ASTOLPHO LOPES ${ }^{1}$, \\ EDIRLEY GUIMARÃES DE SOUZA ${ }^{1}$, LUIZ CLÁUDIO REEBERG STANGANELLI ${ }^{1}$ \\ ${ }^{1}$ Londrina State University, Londrina, Brazil \\ ${ }^{2}$ Western Paraná State University, Marechal Cândido Rondon, Brazil
}

\begin{abstract}
Purpose. The objective was to verify the effects of equal training applied during a training macrocycle on the physiological indicators, body composition measures, and explosiveness of recreational runners.

Methods. The sample consisted of 12 recreational runners, 7 men and 5 women (aged $42.3 \pm 5.4$ and $39.2 \pm 6.5$ years, respectively). The macrocycle was 12 weeks long. In pre- and post-training period, the subjects were submitted to anthropometric measurements and tests to determine the maximal oxygen consumption, velocity associated with maximal oxygen consumption, running economy, and strength of the lower limbs.
\end{abstract}

Results. Significant adaptations were verified between the indicators of countermovement jump $(p<0.036)$ and maximal oxygen consumption $(p<0.003)$. Moderate correlations were observed between weight and countermovement jump ( $\rho=-0.664)$, weight and drop jump $(\rho=-0.657)$, body fat percentage and drop jump $(\rho=-0.636)$. Trivial effect sizes were reported for all variables.

Conclusions. The results evidenced that the loads applied were sufficient only to generate significant adaptations in the muscle strength and aerobic power of the subjects.

Key words: explosiveness, athletic performance, training program, runners

\section{Introduction}

Regular sports practice provides the individual with numerous benefits to physical and mental health, as well as greater social integration. As a result, the number of people who opt to practice a sport to improve their quality of life is increasing [1]. Recreational running has been gaining preference because of many factors: it can be performed alone or in a group, is a low-cost sport, and requires little knowledge or experience to get started [2].

The growth in the number of individuals who choose to practice this modality is motivated, initially, by several reasons, such as health improvement, social integration, well-being, or simply the desire for participation in a competitive activity. Regarding participation in competitions, a good position guarantees prizes, financial support, social recognition, and has been promoted by the growing number of competitions [3].

Considering the context of sports training, it is known that performance is determined by several aspects established through the interaction between physiological and environmental factors [4]. With respect to street races, maximal oxygen consumption $\left(\mathrm{VO}_{2} \mathrm{max}\right)$, velocity corresponding to $\mathrm{VO}_{2} \max \left(\mathrm{vVO}_{2} \max \right)$, and blood lactate threshold responses are among the best physiological indicators related to performance [5], including neuromuscular modulation [6, 7]. Studies have shown that neuromuscular variables are able to significantly interfere with performance, promote delayed fatigue and improvement in anaerobic capacity and maximum speed [8], and impact on running economy (RE) through the reduction in oxygen consump-

Correspondence address: Karina Alves da Silva, Universidade Estadual do Oeste do Paraná, Marechal Cândido Rondon, 85960-000, Paraná, Brazil, e-mail: Karina.silva15@Unioeste.br

Received: January 9, 2019

Accepted for publication: July 18, 2019

Citation: da Silva KA, Lopes JA, de Souza EG, Stanganelli LCR. Effect of a training macrocycle on physiological indicators, body composition, and explosiveness among recreational runners. Hum Mov. 2020;21(1):49-56; https://doi.org/10.5114/ hm.2020.88153. 
K.A. da Silva, J.A. Lopes, E.G. de Souza, L.C.R. Stanganelli, Training macrocycle in recreational runners

tion at a given running speed [5]. Other studies show that the combination of high-intensity and short-duration training models [9] with low-intensity and long-duration and interval intensity protocols $[10,11]$ improves $\mathrm{VO}_{2} \max$.

Thus, although the literature has presented research that relates the adaptations in the variables of performance in runners to the type of training applied [12], the verification of the impact of a single training macrocycle with loads, volume, and intensity for men and women is still a lacune in literature.

As the number of participants and competitions increases [3], many runners are currently involved in running clubs where it is common to prescribe training with loads, volume, and intensity equal for men and women. Thus, we asked the following questions: What are the effects of the same training program prescribed by running clubs on the physiological indices and explosiveness of recreational runners? Will men and women be able to achieve significant adaptations of performance?

With this study, we hope to provide technicians and coaches of running clubs with better information on the effects of a single training program on the performance of men and women so that they can structure more appropriate training loads for athletes aiming for maximum competitive performance in the modality [13].

In order to investigate this gap in literature, we established the objective to verify the effects of equal training applied during a training macrocycle on the physiological indicators, body composition measurements, and explosiveness in recreational runners.

\section{Material and methods}

\section{Participants}

Overall, 12 moderately trained recreational runners were included in the study: 7 men $(42.3 \pm 5.4$ years; $77.2 \pm 13.1 \mathrm{~kg} ; 174 \pm 0.0 \mathrm{~cm})$ and 5 women $(39.2 \pm 6.5$ years; $61.7 \pm 4.9 \mathrm{~kg} ; 167 \pm 0.0 \mathrm{~cm})$, members of a street racing sports club.

As inclusion criteria, individuals were required to present a minimum 1 year of experience in street races, attendance in at least 3 race training sessions per week, a running pace of $5-6 \mathrm{~min} / \mathrm{km}$ for $10-\mathrm{km}$ races, and participation in $\geqslant 80 \%$ of the prescribed sessions in the macrocycle. All participants were informed about the study procedures.

\section{Procedures}

Data were collected during 2 visits, with a minimum interval of 24 hours, which preceded the pre- and posttraining periods; both occurred in the sports sciences laboratory of the Londrina State University.

In their first visit, the participants were asked to complete an anamnesis form containing personal information and information about the training program. Subsequently, anthropometric measures were evaluated, and a maximal treadmill test was carried out to identify $\mathrm{VO}_{2}$ max values. In their second visit, the subjects performed a countermovement jump (CMJ) and a drop jump (DJ). Finally, to determine RE values, they completed a submaximal test on a treadmill.

It should be emphasized that the training program prescribed by a sports consultant was the same for all participants of the study and lasted for 12 weeks. Throughout this period, the researcher had access to the training conducted.

During the evaluation period, the subjects were advised to avoid consuming caffeine-based beverages and foods and not to engage in vigorous physical activity for at least 24 hours prior to testing, as well as to remain fasted for at least 2 hours before the evaluations.

With regard to anthropometric evaluation, to obtain body weight, digital scales (Urano Ps 180) with a capacity of $180 \mathrm{~kg}$ were used. The subjects were instructed to stand on the platform barefoot, wearing minimal clothing, with their backs to the measuring scale, keeping their body erect, legs slightly apart, and eyes fixed to the front.

Height was determined with a wooden stadiometer with a scale accurate to $0.1 \mathrm{~cm}$. The participants stood barefoot on the ground, erect, with their feet together. It was advised that they remained in apnoea and maintained the position of the head in the Frankfurt plane.

To establish the thickness of the skinfolds, we used a Cescorf calliper, with a precision of $1.0 \mathrm{~mm}$, and adopted the 7-fold protocol proposed by Jackson and Pollock [14]. Three measurements were taken for each point and the median value obtained at each point was recorded. Next, to estimate the body fat percentage (\%BF), the formula of Siri was applied [15].

$\mathrm{VO}_{2}$ max was determined by performing an incremental maximal stress test on a treadmill (Inbramed, model 10500), with the use of a $K 4 b^{2}$ spirometer (Cosmed $^{\circledast}$, Italy). A ramp protocol [16] was adopted, consisting of a warm-up at $8 \mathrm{~km} \cdot \mathrm{h}^{-1}$ for 3 minutes, with speed increments of $1 \mathrm{~km} \cdot \mathrm{h}^{-1}$ per each subsequent minute until the subject was unable to main- 
tain the exercise speed. It is emphasized that the participants were verbally encouraged to run to maximum voluntary exhaustion.

Heart rate was monitored throughout the test by using a Polar Team System 2 monitor. At the end of each minute, the value was reported referring to the subjective perception of effort in accordance with the Borg scale [17] (6-20 points).

Observation of a plateau in $\mathrm{VO}_{2}$ max or 2 of the following indicators were established as the criteria for $\mathrm{VO}_{2}$ max selection: $90 \%$ maximum heart rate range based on the Karvonen equation (220 - age); subjective perception of effort $\geq 19$ in the Borg scale; and the ratio of respiratory rate greater than 1 .

The value of $\mathrm{vVO}_{2} \max$ was considered to be the lowest exercise intensity where $\mathrm{VO}_{2}$ max occurred and was maintained for at least 1 minute [18].

$\mathrm{RE}$ was determined on a treadmill (Inbramed, model 10500), with the use of a K4b $\mathrm{b}^{2}$ spirometer (Cosmed $^{\circledR}$, Italy). A running protocol was applied composed of a 2-minute warm-up at $8 \mathrm{~km} \cdot \mathrm{h}^{-1}$, followed by a 15 -minute run at an intensity of $10 \mathrm{~km} \cdot \mathrm{h}^{-1}$. To identify RE, $\mathrm{O}_{2}$ consumption and the respiratory exchange ratio were adopted between the $10^{\text {th }}$ and $14^{\text {th }}$ minutes of the test [19].

To determine the capacity explosiveness of the lower limbs, the CMJ technique [20] was applied. Three vertical upward thrust attempts were performed, with a 30-second interval between them, on a jump plate coupled to the Multsprint 1.20 software from the Multsprint Kit (Hidrofit, Brazil). The explosive strength was expressed as height in centimetres, with the consideration of the greatest height obtained.

Contact time was established with DJ [20]. The subjects performed 3 vertical thrust attempts, with a 30-second interval between them, from a platform with a height of $40 \mathrm{~cm} ; 2$ bars with sensors (Sys Jump, Systware $^{\circledast}$, Brazil) were used to record the times of contact (ms). The shortest contact time was adopted to determine the explosive potential.

\section{Statistical analysis}

Data were described as the values of mean, standard deviation, raw delta, and percentage delta. Normality was verified with the Shapiro-Wilk test.

The paired $t$-test was applied to detect the possible pre- and post-training differences for the variables of body composition, physiological indicators, and explosiveness.

Spearman's correlation was used between the preand post-training moments by means of the percentage delta analysis of the variables evaluated.
To identify possible differences between the moments in the indicators evaluated, the paired $t$-test was applied.

To interpret the correlation values, the criterion of Pestana and Gageiro [21] was adopted, which determines that correlation values $<0.60$ correspond to a very low correlation, those of $0.60-0.69$ to low correlation, those of 0.70-0.79 to moderate correlation, those of 0.80-0.89 to good correlation, and those of $0.90-1$ to very good correlation. Statistical significance of the results was accepted at $p<0.05$.

The formula proposed by Rhea [22] served o calculate the effect size $(E S)$. Values $<0.5$ were considered as trivial $E S$, those of $0.5-1.25$ as small $E S$, those of $1.25-1.9$ as moderate $E S$, and those $\geq 2.0$ as high ES. The analyses were performed with SPSS software, version 20.0.

\section{Ethical approval}

The research related to human use has complied with all the relevant national regulations and institutional policies, has followed the tenets of the Declaration of Helsinki, and has been approved by the Committee of Ethics and Research on Human Beings of Londrina State University under opinion No. 1.534.572/2016.

\section{Informed consent}

Informed consent has been obtained from all individuals included in this study.

\section{Results}

The training volume and description of the training sessions during the 12-week study are provided in Tables 1 and 2, respectively.

Table 3 presents the results of the comparisons of the variables: weight, $\% \mathrm{BF}, \mathrm{CMJ}, \mathrm{DJ}, \mathrm{VO}_{2} \mathrm{max}, \mathrm{vVO}_{2} \max$, and RE at the pre- and post-training moments for the total sample. Statistically significant differences $(p<$ $0.05)$ were observed only for $\mathrm{CMJ}(0.036)$ and $\mathrm{VO}_{2} \max$ (0.003).

Table 4 shows the analysis of raw and percentage delta values before and after 12 weeks of training. A significant increase was evidenced in the differences between the means and standard deviations of the variables of $\mathrm{CMJ}$ and $\mathrm{VO}_{2}$ max.

With regard to the values of the correlations, the results presented in Table 5 demonstrate that there was a moderate correlation between the following indicators: weight and CMJ $(\rho=-0.664)$, weight and DJ $(\rho=0.657)$, and $\%$ BF and DJ $(\rho=0.636)$. In turn, 


\section{HUMAN MOVEMENT}

K.A. da Silva, J.A. Lopes, E.G. de Souza, L.C.R. Stanganelli, Training macrocycle in recreational runners

Table 1. Training volume of the group during the 12-week study

\begin{tabular}{ccccccc}
\hline Microcycle & Wednesday $(\mathrm{km})$ & Thursday $(\mathrm{km})$ & Friday $(\mathrm{km})$ & Saturday $(\mathrm{km})$ & Monday $(\mathrm{km})$ & Total $(\mathrm{km})$ \\
\hline 1 & 10 & Rest & Rest & 14 & 8 & 32 \\
2 & 8 & Rest & 12 & 14 & 10 & 44 \\
3 & 8 & Rest & Rest & 17 & 10 & 35 \\
4 & 8 & Rest & 12 & 18 & 10 & 48 \\
5 & 6 & Rest & 14 & 20 & 10 & 50 \\
6 & 16 & Rest & 10 & 30 & 14 & 70 \\
7 & 10 & Rest & 10 & 16 & 10 & 46 \\
8 & 8 & 17 & Rest & 12 & 10 & 47 \\
9 & 8 & Rest & Rest & 18 & 10 & 36 \\
10 & 8 & Rest & Rest & 26 & 10 & 44 \\
11 & 6 & Rest & 8 & 15 & 10 & 39 \\
12 & 10 & Rest & 20 & 36 & 12 & 78 \\
\hline
\end{tabular}

Table 2. Characteristics of the training sessions during the 12-week study

\begin{tabular}{|c|c|c|c|c|c|c|c|c|c|c|}
\hline Microcycle & $\begin{array}{c}\text { No. of } \\
\text { sessions }\end{array}$ & $\begin{array}{c}\text { Method } \\
\text { in session } \\
1\end{array}$ & $\begin{array}{c}\text { Method } \\
\text { in session } \\
2\end{array}$ & $\begin{array}{c}\text { Method } \\
\text { in session } \\
3\end{array}$ & $\begin{array}{c}\text { Method } \\
\text { in session } \\
4\end{array}$ & Intensity & $\begin{array}{c}\text { Lowest } \\
\text { weekly } \\
\text { volume } \\
(\mathrm{km})\end{array}$ & $\begin{array}{c}\text { Highest } \\
\text { weekly } \\
\text { volume } \\
(\mathrm{km})\end{array}$ & $\begin{array}{l}\text { Mean } \\
\text { training } \\
\text { volume } \\
(\mathrm{km})\end{array}$ & $\begin{array}{c}\text { Total } \\
\text { training } \\
\text { load } \\
(\mathrm{km})\end{array}$ \\
\hline 1 & 3 & $\mathrm{EC}$ & $\mathrm{EC}$ & VA & - & $\mathrm{LO} / \mathrm{MO} / \mathrm{HI}$ & 8 & 14 & 10.6 & 32 \\
\hline 2 & 4 & $\mathrm{EC}$ & INT + VA & $\mathrm{EC}$ & $\mathrm{EC}$ & $\mathrm{LO} / \mathrm{MO}$ & 8 & 14 & 11 & 44 \\
\hline 3 & 3 & INT & $\mathrm{EC}$ & INT & _- & $\mathrm{MO} / \mathrm{HI}$ & 8 & 17 & 11.6 & 35 \\
\hline 4 & 4 & INT + VA & $\mathrm{EC}$ & $\mathrm{EC}$ & VA & $\mathrm{LO} / \mathrm{MO} / \mathrm{HI}$ & 8 & 18 & 12 & 48 \\
\hline 5 & 4 & PRO & $\mathrm{EC}$ & $\mathrm{EC}$ & PRO & LO/MO/HI & 6 & 20 & 12.5 & 50 \\
\hline 6 & 4 & $\mathrm{PRO}+\mathrm{EC}$ & $\mathrm{EC}$ & $\mathrm{EC}$ & INT & $\mathrm{LO} / \mathrm{MO}$ & 10 & 30 & 17.5 & 70 \\
\hline 7 & 4 & VA & $\mathrm{EC}$ & PRO & $\mathrm{EC}$ & $\mathrm{LO} / \mathrm{MO} / \mathrm{HI}$ & 10 & 16 & 11.5 & 46 \\
\hline 8 & 4 & VA & $\mathrm{EC}$ & $\mathrm{EC}$ & INT & $\mathrm{LO} / \mathrm{MO}$ & 8 & 17 & 11.7 & 47 \\
\hline 9 & 3 & PLI & - & $\mathrm{EC}$ & $\mathrm{EC}$ & $\mathrm{LO} / \mathrm{MO}$ & 8 & 18 & 12 & 36 \\
\hline 10 & 3 & INT + VA & - & $\mathrm{EC}+\mathrm{VA}$ & $\mathrm{EC}$ & $\mathrm{LO} / \mathrm{MO}$ & 8 & 26 & 14.6 & 44 \\
\hline 11 & 4 & $\mathrm{EC}$ & $\mathrm{EC}$ & $\mathrm{INT}+\mathrm{PRO}+\mathrm{EC}$ & $\mathrm{EC}$ & $\mathrm{LO} / \mathrm{MO} / \mathrm{HI}$ & 6 & 15 & 9.7 & 39 \\
\hline 12 & 4 & $\mathrm{EC}$ & $\mathrm{EC}$ & EC & $\mathrm{EC}$ & $\mathrm{LO} / \mathrm{MO}$ & 10 & 36 & 19.5 & 78 \\
\hline
\end{tabular}

EC - extensive continuous, INT - interval, PLI - plyometric, PRO - progressive, VA - variational,

HI - high, LO - low, MO - moderate

Table 3. Comparison of performance in the physiological indicators of the total sample of street runners before and after the 12-week training period

\begin{tabular}{lccccc}
\hline \multirow{2}{*}{ Variable } & \multicolumn{3}{c}{ Pre } & \multicolumn{3}{c}{ Post } & \multirow{2}{*}{$p$} \\
\cline { 2 - 5 } & $M$ & $S D$ & $M$ & $S D$ & \\
\hline Weight $(\mathrm{kg})$ & 70.78 & 12.91 & 70.70 & 12.89 & 0.856 \\
\%BF $(\%)$ & 13.94 & 4.61 & 13.84 & 4.43 & 0.733 \\
$\mathrm{CMJ}(\mathrm{cm})$ & 26.78 & 5.80 & 28.40 & 5.31 & 0.036 \\
$\mathrm{DJ}(\mathrm{ms})$ & 0.250 & 0.140 & 0.240 & 0.130 & 0.727 \\
$\mathrm{VO}_{2} \mathrm{max}\left(\mathrm{ml} \cdot \mathrm{kg}^{-1} \cdot \mathrm{min}^{-1}\right)$ & 48.11 & 2.84 & 49.23 & 2.83 & 0.003 \\
$\mathrm{vVO}_{2} \mathrm{max}\left(\mathrm{km}^{-1} \cdot \mathrm{h}^{-1}\right)$ & 15.75 & 1.22 & 15.33 & 1.07 & 0.096 \\
$\mathrm{RE}\left(\mathrm{ml} \cdot \mathrm{kg}^{-1} \cdot \mathrm{min}^{-1}\right)$ & 36.94 & 2.28 & 37.71 & 2.35 & 0.052 \\
\hline
\end{tabular}

\%BF - percentage of body fat, $\mathrm{CMJ}$ - countermovement jump, DJ - drop jump, $\mathrm{VO}_{2} \mathrm{max}$ - maximal oxygen consumption, $\mathrm{vVO}_{2} \max$ - running velocity associated with maximal oxygen consumption, $\mathrm{RE}$ - running economy,

$M$ - mean, $S D$ - standard deviation 
Table 4. Results of evaluations of the total sample before and after the 12-week training period in raw delta and percentage delta, presented as means and standard deviations

\begin{tabular}{|c|c|c|c|c|c|c|c|c|c|}
\hline \multirow{2}{*}{ Variable } & \multicolumn{4}{|c|}{ Raw delta } & \multicolumn{4}{|c|}{ Delta (\%) } & \multirow{2}{*}{$p$} \\
\hline & $M$ & $S D$ & Min & Max & $M$ & $S D$ & Min & Max & \\
\hline Weight (kg) & -0.08 & 1.4 & -2.9 & 1.6 & -0.1 & 1.9 & -3.1 & 2.1 & 0.856 \\
\hline$\%$ BF (\%) & -0.09 & 0.92 & -2.06 & 1.35 & -0.3 & 6.5 & -9.7 & 11.9 & 0.733 \\
\hline $\mathrm{CMJ}(\mathrm{cm})$ & 1.62 & 2.34 & -1 & 7.5 & 6.9 & 9.5 & -3.3 & 30 & 0.036 \\
\hline $\mathrm{DJ}(\mathrm{ms})$ & -0.01 & 0.06 & -0.17 & 0.07 & -0.2 & 15.2 & -33.2 & 28 & 0.727 \\
\hline $\mathrm{VO}_{2} \max \left(\mathrm{ml} \cdot \mathrm{kg}^{-1} \cdot \mathrm{min}^{-1}\right)$ & 1.12 & 1.02 & -0.46 & 2.75 & 2.36 & 2.18 & -0.99 & 6.2 & 0.003 \\
\hline $\mathrm{vVO}_{2} \max \left(\mathrm{km} \cdot \mathrm{h}^{-1}\right)$ & -0.41 & 0.79 & -2 & 1 & -2.4 & 4.9 & -11 & 7.1 & 0.096 \\
\hline $\mathrm{RE}\left(\mathrm{ml} \cdot \mathrm{kg}^{-1} \cdot \mathrm{min}^{-1}\right)$ & 0.77 & 1.22 & -1.66 & 2.8 & 2.1 & 3.3 & -4.1 & 8.1 & 0.052 \\
\hline
\end{tabular}

$\% \mathrm{BF}$ - percentage of body fat, CMJ - countermovement jump, DJ - drop jump, $\mathrm{VO}_{2} \mathrm{max}$ - maximal oxygen consumption, $\mathrm{vVO}_{2} \mathrm{max}$ - running velocity associated with maximal oxygen consumption, RE - running economy,

$M$ - mean, $S D$ - standard deviation

Table 5. Correlation of the physiological and muscular strength indicators of the total sample of street runners in the pre- and post-training period

\begin{tabular}{|c|c|c|c|c|c|c|c|}
\hline \multirow{2}{*}{\multicolumn{2}{|c|}{ Variable }} & \multicolumn{6}{|c|}{ Delta (\%) } \\
\hline & & $\% \mathrm{BF}$ & $\mathrm{CMJ}$ & DJ & $\mathrm{VO}_{2} \max$ & $\mathrm{vVO}_{2} \max$ & $\mathrm{RE}$ \\
\hline \multirow{6}{*}{ 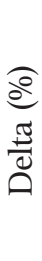 } & Weight & 0.406 & $-0.664^{*}$ & $0.657^{*}$ & 0.154 & 0.022 & -0.343 \\
\hline & $\% B F$ & & $-0.769 * *$ & $0.636^{*}$ & -0.196 & -0.254 & -0.308 \\
\hline & $\mathrm{CMJ}$ & & & $-0.713 * *$ & 0.217 & 0.285 & 0.469 \\
\hline & DJ & & & & 0.028 & -0.456 & -0.552 \\
\hline & $\mathrm{VO}_{2} \max$ & & & & & $0.576 *$ & 0.021 \\
\hline & $\mathrm{vVO}_{2} \max$ & & & & & & -0.060 \\
\hline
\end{tabular}

\%BF - percentage of body fat, CMJ - countermovement jump, DJ - drop jump, $\mathrm{VO}_{2} \mathrm{max}$ - maximal oxygen consumption, $\mathrm{vVO}_{2} \mathrm{max}$ - running velocity associated with maximal oxygen consumption, RE - running economy

* moderate correlations

** high correlations

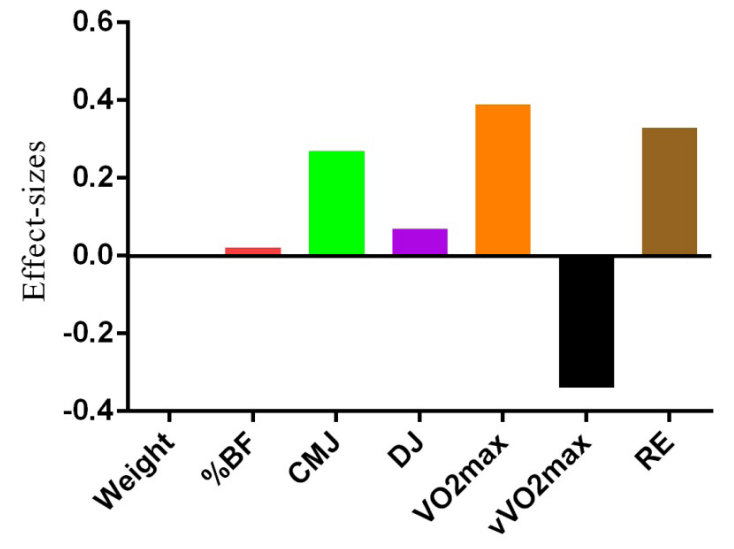

\%BF - percentage of body fat, CMJ - countermovement jump, DJ - drop jump, $\mathrm{VO}_{2} \mathrm{max}$ - maximal oxygen consumption, $\mathrm{VVO}_{2} \mathrm{max}$ - running velocity associated with maximal oxygen consumption, $\mathrm{RE}$ - running economy

Figure 1. Post-training effect sizes in the physiological indicators and muscle strength among runners a high correlation was observed between $\% \mathrm{BF}$ and $\mathrm{CMJ}$ $(\rho=-0.769)$ and between CMJ and DJ $(\rho=-0.713)$.

In Figure 1, a trivial increase in $E S$ can be observed in all indicators evaluated, except for weight, which did not demonstrate any variation between the moments.

\section{Discussion}

The study investigated the effects of equal training applied during a training macrocycle on the physiological indicators, body composition measures, and explosiveness of recreational runners.

The information obtained herein allowed to verify how training loads were prescribed by the trainers and to identify whether the responses resulting from the training process were sufficient to present satisfactory improvement in the performance of the analysed variables.

The results demonstrated that the applied loads were sufficient to generate significant adaptations only in the $\mathrm{CMJ}$ and $\mathrm{VO}_{2}$ max indicators. Moderate correla- 
K.A. da Silva, J.A. Lopes, E.G. de Souza, L.C.R. Stanganelli, Training macrocycle in recreational runners

tion coefficients were found between $\% \mathrm{BF}$ and CMJ, DJ and CMJ. On the other hand, in general, the ES magnitude for physiological indicators and explosiveness was trivial.

As for the variables that presented significant responses, an increase of $6.9 \%$ was observed in the height obtained in the CMJ jump. This value was lower than the $7.2 \%$ reported by Crivoi do Carmo [16] when 2 plyometric training sessions were included in male runners over a period of 8 weeks, and also lower than the $8.9 \%$ evidenced by Ramírez-Campillo et al. [23], who included the same number of plyometric sessions for 8 weeks among runners of both sexes. Thus, it is possible that the runners presented a lower performance in the CMJ jump when compared with subjects of the other studies [16, 23] owing to the low number of sessions aimed at the development of this capacity.

Positive improvements in explosiveness may be obtained from the application of maximum strength and explosive strength training models; the inversely proportional correlation values between CDJ and DJ as evidenced by the literature is related to parameters of loss or gain of explosiveness $[11,24]$. Thus, the greater the gain in the height obtained in the CMJ jump and the shorter the contact time in the DJ, the greater the explosiveness increment. When analysing the training macrocycle of the runners in the present study, it was verified that there were few sessions aimed at the development of explosive force, without a structured frequency, which could generate returns lower than expected in explosiveness, although the subjects presented positive responses in this variable. This demonstrates that even with little time dedicated to the development of this physical capacity during the macrocycle, it seems possible to observe a positive adaptation, which is important for maintaining levels of training and consequent performance in competitions.

Considering the $\mathrm{VO}_{2} \max$, the literature indicates that both the combination of high-intensity and lowvolume loads [9] and the application of low-intensity loads and interval protocols $[10,11]$ may contribute to increases in the values of this variable. The increase to $48.11 \mathrm{ml} \cdot \mathrm{kg}^{-1} \cdot \mathrm{min}^{-1}$ in the post-training $\mathrm{VO}_{2}$ max values generated a significant rise of $2.36 \%$ in relation to the first evaluation, demonstrating that there was a small improvement in the aerobic fitness of the subjects of the present study. Although they presented a certain amount of experience in street races, the $\mathrm{VO}_{2}$ max of the individuals evaluated was lower than that among the participants of the study by Iaia et al. [25], men with a mean age of 33.5 years and $\mathrm{VO}_{2}$ max of approximately $54.80 \mathrm{ml} \cdot \mathrm{kg}^{-1} \cdot \mathrm{min}^{-1}$; of the study by
Zatoń and Michalik [26], men and women with a mean age of 34 years and $\mathrm{VO}_{2} \max$ of $51.98 \mathrm{ml} \cdot \mathrm{kg}^{-1} \cdot \mathrm{min}^{-1}$; and of the study by Manou et al. [27], 25 male adolescents aged 13-16.1 years with training experience time with soccer of $6.7 \pm 1.8$ years, who presented an increase of $6.65 \%$ in $\mathrm{VO}_{2}$ max values after 2 preseason periods. Given that approximately $75 \%$ of the training sessions of the macrocycle were composed of low-intensity and high-volume loads, the improvement observed in aerobic fitness could be related to the fact that the participants of the present research were more prone to sensitive cardiorespiratory adaptations as a function of training status when compared with runners with higher levels of performance [28], such as the subjects of the above-mentioned studies.

It is important to note that when analysing the training program (Table 2), one can observe an exponential increase in training volume of approximately 50\% from the penultimate to the last microcycle. Regardless of how the training loads were prescribed (the researcher did not control this), there was a positive and significant response for the $\mathrm{VO}_{2}$ max variable, which is important for the maintenance of training levels and also possible improvements in competitive performance, although other variables, such as explosiveness and RE, may influence the end result.

With regard to the adaptations observed in the RE variable after the training period, there was a $2.1 \%$ increase in the energy demand of the subjects. This index is very close to the established range of $2.2-2.6 \%$, considered significant for RE improvement [28]. Thus, despite not finding significant values for this variable, several studies have shown that neuromuscular factors can positively impact RE. Recent research reinforces that positive responses in RE, along with increased explosiveness, improve the time of contact with the ground during the race and, consequently, demand less energy expenditure on the part of the athletes.

With respect to the significant correlations, the moderate values observed between the $\% \mathrm{BF}$ and CMJ variables $(r$ : -0.769$)$ may explain why the values of body composition correlated negatively with performance in the motor test. Considering that many sports show correlations between performance variables and somatic features [29], the negative overload generated by the increase in body composition values affects the execution of the motor gestures and, consequently, jumping impulse force [30]. The correlation between CMJ and DJ ( $r$ : 0.713$)$ with increases in explosiveness are verified from the greater height in the CMJ jump when analysing the performance of the subjects in the DJ jump; the highest values of this ability 
are related to the shorter contact times with the jumping performance, which may have a relation with the time of ground contact during the execution of strides in races.

When analysing the magnitudes of effect on the physiological indicators and other variables analysed, although significant alterations were observed for the CMJ jump and $\mathrm{VO}_{2}$ max, they had a trivial effect on performance, with increments of 0.27 and 0.16 , respectively. Berryman et al. [31] found similar values for the same variables. Trivial effects of 0.25 for the CMJ jump and 0.20 for $\mathrm{VO}_{2}$ max were verified after an intervention period of 8 weeks of training with weights for male runners, whereas effects of 0.52 for the CMJ jump and 0.03 for $\mathrm{VO}_{2}$ max were evidenced with the same subjects after the application of a plyometric protocol over an 8-week period. This information indicates that the stimuli generated by the applied training loads were not sufficient to induce an effective contribution to improvement in the evaluated parameters.

Although the results of the tests imply that the loads used in the macrocycle generated small responses in the evaluated variables, it should be taken into account that for the studied sample, the changes resulting from the training, although small, enabled the individuals to complete higher running mileage than they had usually performed, without suffering detriments from the training loads applied.

Thus, it is recommended that future studies seek to verify the impact of a training macrocycle on alterations in physiological indices and explosiveness with a larger number of runners and also to compare the periodization of macrocycles prescribed by different groups or consultants in order to produce a reference for better identification of the distribution of loads and impact generated on the performance of recreational runners.

\section{Conclusions}

The study allows to conclude that the loads applied in the macrocycle were sufficient only to generate significant adaptations in the explosiveness and aerobic power of the subjects. In addition, moderate correlation coefficients were found. It was verified that the magnitude of $E S$ was trivial for all the indicators evaluated.

\section{Disclosure statement}

No author has any financial interest or received any financial benefit from this research.

\section{Conflict of interest}

The authors state no conflict of interest.

\section{References}

1. Scheerder J, Breedveld K, Borgers J. Running across Europe: the rise and size of one of the largest sport markets. London: Palgrave Macmillan; 2015.

2. Shipway R, Holloway I. Running free: embracing a healthy lifestyle through distance running. Perspect Public Health. 2010;130(6):270-276; doi: 10.1177/ 1757913910379191.

3. Salgado JVV, Chacon-Mikahil MPT. Street race: analyses of the growth of the number of competitions and practitioners [in Potruguese]. Conexões. 2006;4(1):90-99.

4. Bray MS, Hagberg JM, Pérusse L, Rankinen T, Roth SM, Wolfarth B, et al. The human gene map for performance and health-related fitness phenotypes: the 2006-2007 update. Med Sci Sports Exerc. 2009;41(1):35-73; doi: 10.1249/mss.0b013e3181844179.

5. Hoff J, Gran A, Helgerud J. Maximal strength training improves aerobic endurance performance. Scand J Med Sci Sports. 2002;12(5):288-295; doi: 10.1034/j.16000838.2002.01140.x.

6. Mikkola J, Rusko H, Nummela A, Pollari T, Häkkinen K. Concurrent endurance and explosive type strength training improves neuromuscular and anaerobic characteristics in young distance runners. Int J Sports Med. 2007;28(7):602-611; doi: 10.1055/s-2007-964849.

7. Taipale RS, Mikkola J, Nummela A, Vesterinen V, Capostagno B, Walker S, et al. Strength training in endurance runners. Int J Sports Med. 2010;31(7):468-476; doi: 10.1055/s-0029-1243639.

8. Rønnestad BR, Mujika I. Optimizing strength training for running and cycling endurance performance: a review. Scand J Med Sci Sports. 2014;24(4):603-612; doi: 10.1111/sms.12104.

9. Gibala MJ, Little JP, Macdonald MJ, Hawley JA. Physiological adaptations to low-volume, high-intensity interval training in health and disease. J Physiol. 2012; 590(5):1077-1084; doi: 10.1113/jphysiol.2011.224725.

10. Helgerud J, Høydal K, Wang E, Karlsen T, Berg P, Bjerkaas M, et al. Aerobic high-intensity intervals improve $\mathrm{VO}_{2 \max }$ more than moderate training. Med Sci Sports Exerc. 2007;39(4):665-671; doi: 10.1249/mss. 0b013e3180304570.

11. Gormley SE, Swain DP, High SR, Spina RJ, Downling EA, Kotipalli US, et al. Effect of intensity of aerobic training on $\mathrm{VO}_{2 \max }$. Med Sci Sports Exerc. 2008;40(7):13361343; doi: 10.1249/MSS.0b013e31816c4839.

12. Milanović Z, Sporiš G, Weston M. Effectiveness of highintensity interval training (HIT) and continuous endurance training for $\mathrm{VO} 2 \mathrm{max}$ improvements: a systematic review and meta-analysis of controlled trials. Sports Med. 2015;45(10):1469-1481; doi: 10.1007/s40279015-0365-0.

13. Roschel H, Tricoli V, Ugrinowitsch C. Physical training: scientific and practical considerations [in Portuguese]. 


\section{HUMAN MOVEMENT}

K.A. da Silva, J.A. Lopes, E.G. de Souza, L.C.R. Stanganelli, Training macrocycle in recreational runners

Rev Bras Educ Fis Esporte. 2011;25(1):53-65; doi: 10.1590/s1807-55092011000500007.

14. Jackson AS, Pollock ML. Generalized equations for predicting body density of men. Br J Nutr. 1978;40(3):497504; doi: 10.1079/bjn19780152.

15. Siri WE. Body composition from fluid spaces and density. In: Brozek J, Henschel A (eds.), Techniques for measuring body composition. Washington DC: National Academy of Sciences; 1961; 223-244.

16. Crivoi do Carmo E. Effect of race economy on race strategy applied during a $10-\mathrm{km}$ race (doctoral thesis). São Paulo: University of São Paulo; 2014.

17. Borg GAV. Scales for pain and perceived effort [in Portuguese]. São Paulo: Manole; 2000.

18. Billat VL, Flechet B, Petit B, Muriaux G, Koralsztein JP. Interval training at VO2max: effects on aerobic performance and overtraining markers. Med Sci Sports Exerc. 1999;31(1):156-163; doi: 10.1097/00005768199901000-00024.

19. Albracht K, Arampatzis A. Exercise-induced changes in triceps surae tendon stiffness and muscle strength affect running economy in humans. Eur J Appl Physiol. 2013; 113(6):1605-1615; doi: 10.1007/s00421-012-2585-4.

20. Bosco C, Kellis S. Methods for evaluating physiological properties of different track and field events [in Italian]. Atletica Studi. 1993;6(6):361-371.

21. Pestana MH, Gageiro JG. Data analysis for social sciences: the complementarity of SPSS [in Portuguese], $3^{\text {rd }}$ ed. Lisboa: Silabo; 2003.

22. Rhea MR. Determining the magnitude of treatment effects in strength training research through the use of the effect size. J Strength Cond Res. 2004;18(1):918920; doi: 10.1519/14403.1.

23. Ramírez-Campillo R, Álvarez C, Henríquez-Olguín C, Baez EB, Martínez C, Andrade DC, et al. Effects of plyometric training on endurance and explosive strength performance in competitive middle- and long-distance runners. J Strength Cond Res. 2014;28(1):97-104; doi: 10.1519/JSC.0b013e3182a1f44c.

24. Sedano S, Marín PJ, Cuadrado G, Redondo JC. Concurrent training in elite male runners: the influence of strength versus muscular endurance training on performance outcomes. J Strength Cond Res. 2013;27(9): 2433-2443; doi: 10.1519/JSC.0b013e318280cc26.

25. Iaia FM, Thomassen M, Kolding H, Gunnarsson T, Wendell J, Rostgaard T, et al. Reduced volume but increased training intensity elevates muscle $\mathrm{Na}+-\mathrm{K}+$ pump alpha1-subunit and NHE1 expression as well as short-term work capacity in humans. Am J Physiol Regul Integr Comp Physiol. 2008;294(3):R966-R974; doi: 10.1152/ajpregu.00666.2007.

26. Zatoń M, Michalik K. Effects of interval training-based glycolytic capacity on physical fitness in recreational long-distance runners. Hum Mov. 2015;16(2):71-77; doi: 10.1515/humo-2015-0029.

27. Manou V, Dalamitros AA, Kellis S. Variations in important aerobic fitness parameters and physical char- acteristics during two consecutive preseason periods in adolescent soccer players. Hum Mov. 2018;19(2):7581; doi: 10.5114/hm.2018.74062.

28. Barnes KR, Kilding AE. Running economy: measurement, norms, and determining factors. Sports Med Open. 2015;1:8; doi: 10.1186/s40798-015-0007-y.

29. Eksterowicz J, Napierała M, Żukow W. How the Kenyan runner's body structure affects sports results. Hum Mov. 2016;17(1):8-14; doi: 10.1515/humo-2016-0002.

30. Markovic G, Mikulic P. Neuro-musculoskeletal and performance adaptations to lower-extremity plyometric training. Sports Med. 2010;40(10):859-895; doi: 10.2165/11318370-000000000-00000.

31. Berryman N, Maurel DB, Bosquet L. Effect of plyometric vs. dynamic weight training on the energy cost of running. J Strength Cond Res. 2010;24(7):1818-1825; doi: 10.1519/JSC.0b013e3181def1f5. 